\title{
ISOMORPHISMS OF FUNCTION ALGEBRAS AND ALGEBRAS OF ANALYTIC FUNCTIONS
}

\author{
BY \\ BRUCE LUND
}

\begin{abstract}
Let $R$ be a finite open Riemann surface with analytic boundary $\Gamma$. Set $\bar{R}=R \cup \Gamma$ and define $A(R)=\{f \in C(\bar{R}): f$ is analytic on $R$ \}. Conditions are given on a function algebra $A$ on a compact Hausdorff space $X$ which imply that $A$ is isomorphic to a subalgebra of $A(R)$ of finite codimension.
\end{abstract}

$\$ 1$ Introduction. Let $R$ be a connected finite open Riemann surface with analytic boundary $\Gamma$ and set $\bar{R}=R \cup \Gamma$. Let $C(\bar{R})$ denote the complex-valued continuous functions on $\bar{R}$. We define $A(R)=\{f \in C(\bar{R}): f$ is analytic on $R\}$. Then $A(R)$ is a function algebra on $\bar{R}$. The goal of this paper is to give an abstract function algebraic characterization of the subalgebras of finite codimension of the function algebra $A(R)$. This work will employ the results of " $f$-regularity" theory introduced by E. Bishop. The main result of the paper will be related to a theorem of T. Gamelin on embedding a Riemann surface in the maximal ideal space of a function algebra.

\$2 Definitions and statement of results. Throughout this paper $A$ will be a function algebra on a compact Hausdorff space $X$. We list some definitions and facts about function algebras and refer the reader to the references [8] and [18] for details. We let $M_{\mathrm{A}}$ denote the maximal ideal space of $A$ and give $M_{\mathrm{A}}$ the weak-star topology which is inherited from the dual space of $A$. If $f \in A$, then $\hat{f}$ will be the Gelfand transform of $f$. If $f$ is a map from $Y$ to $Z$, then $\# f^{-1}(z)$ denotes the cardinality of the set $f^{-1}(z)$. In particular, if $f \in A$, then $\# \hat{f}^{-1}(s)$ denotes the cardinality of the set $\left\{\sigma \in M_{A}: \hat{f}(\sigma)=s\right\}$.

We next define the notion of an analytic embedding $\Psi$ from $R$ into $M_{A}$. Let $H^{\infty}(R)$ be the set of bounded analytic functions on $R$. Let $W \subset M_{\mathrm{A}}$. If there is a continuous mapping $\Psi$ of $R$ onto $W$ (where $W$ has the relative topology of $M_{A}$ ) such that $\tilde{f}(z)=\hat{f} \circ \Psi(z) \in H^{\infty}(R)$ for $f \in A$, then we will call $\Psi$ an analytic mapping from $R$ onto $W$. We will be concerned with analytic maps $\Psi$ for which the set $\{(a, b): a \neq b, \Psi(a)=\Psi(b)\}$ is finite. In this case we will say that $\Psi$ identifies a finite number of points.

AMS (MOS) Subject Classification, Primary 46J10

Key Words and Phrases: function algebra, Gleason part, $f$-regular

Supported in part by NRC (of Canada) grant A8763.

Received by the editors January 4, 1977 and, in revised form, July 29, 1977. 
As further notation we use $T=\{z:|z|=1\}$ and $\Delta=\{z:|z|<1\}$. Our principal result is stated below:

Theorem 1. Let $A$ be a function algebra on $X$. Assume that

(i) There is $W \subset M_{\mathrm{A}}$ and an analytic map $\Psi: R \rightarrow W$ such that $\Psi$ identifies at most a finite number of points.

(ii) $\bar{W} \backslash W=X$

(iii) The set $S=\{g \in A:|g|=1$ on $X\}$ separates points on $X$ and there is $F \in S$, nonconstant on $X$, such that $E=\left\{x \in T: \# \hat{F}^{-1}(x)<\infty\right\}$ contains a set of positive Lebesgue measure in $T$.

Then $\Psi$ extends continuously to $\bar{R}$ and maps $\Gamma$ onto $X$. Let $\tilde{\Psi}: A \rightarrow A(R)$ be defined by $\tilde{\Psi}(f)=\hat{f} \circ \Psi$ and define $A^{\prime}=\tilde{\Psi}(A)$. Then $\tilde{\Psi}$ is an isomorphism of $A$ onto $A^{\prime}$ and as a vector subspace $A^{\prime}$ has finite codimension in $A(R)$. Moreover, $M_{A}=\bar{W}$.

REMARK. Let $f$ be analytic in a neighborhood of $\bar{R}$. If there is an integer $n$ such that $f$ maps $\bar{R}$ onto $\bar{\Delta}$ in an $n$-to-one manner counting multiplicity with $f(\Gamma)=T$, then we will call $f$ a unimodular function. That such functions exist was proved by Ahlfors. Stout [17] showed that the unimodular functions separate points on $\bar{R}$. In light of these remarks, it is clear that $A(R)$ satisfies the hypotheses (i), (ii), (iii) of the theorem above.

For later reference we define the order of a unimodular function $f$ to be $n$ if $f$ is an $n$-to-one (counting multiplicity) map of $\bar{R}$ onto $\bar{\Delta}$.

$\S 3$. Preliminary lemmas. Let the boundary $\Gamma$ of $R$ consist of simple closed analytic curves $\Gamma_{1}, \ldots, \Gamma_{n}$. We call $B \subset R$ a boundary strip if $B$ consists of $n$ pairwise disjoint annular domains $K_{1}, \ldots, K_{n}$ such that $\Gamma_{j} \subset \bar{K}_{j}$ and such that there is a coordinate map $\phi_{j}$ from an annulus $\{z: s<|z|<1\}$ onto $K_{j}$ which extends to map $T$ onto $\Gamma_{j}$.

The lemma below appears to be straightforward; however, a careful proof becomes somewhat detailed. This result does not seem to appear in standard references.

Lemma 1. Let $f: R \rightarrow \mathbb{C}$ be an analytic map. Suppose there is a positive integer $m$ such that $\# f^{-1}(x) \leq m$ for all $x \in f(R)$ and suppose $E=$ $\left\{x \in f(R): \# f^{-1}(x)<m\right\}$ is a discrete set in $f(R)$. Then $f$ is a proper map. (That is, if $L \subset f(R)$ is compact, then $f^{-1}(L)$ is compact in $R$.)

Proof. It suffices to show that if $x_{0} \in f(R)$, then there is a compact neighborhood $N$ of $x_{0}$ such that $f^{-1}(N)$ is compact in $R$. The case where $x_{0} \in f(R) \backslash E$ is straightforward and we consider the case where $x_{0} \in E$. Let $G$ be an open set with compact closure in $R$ such that $f^{-1}\left(x_{0}\right) \subset G$. Pick a disk $V(r)$ about $x_{0}$ of radius $r$ so that $V(r) \subset f(G)$ and $V(r) \cap E=\left\{x_{0}\right\}$. We wish to show that $r$ may be picked so small that $f^{-1}(\overline{V(r)}) \subset \bar{G}$ which will prove the Lemma.

Given a boundary strip $B$ such that $\bar{B} \cap \bar{G}=\varnothing$, it is easy to prove that $r$ may 
be chosen so small that $f^{-1}(V(r)) \subset G \cup B$. Suppose $B$ and $r$ are selected as above. We wish to show that $f^{-1}(V(r)) \cap B=\varnothing$. We suppose the contrary.

Let $V_{0}(r)=V(r) \backslash\left\{x_{0}\right\}$. Then $f$ is an $m$-to one map of $f^{-1}\left(V_{0}(r)\right)$ onto $V_{0}(r)$. It follows that if $f$ is restricted to any component $U$ of $f^{-1}\left(V_{0}(r)\right)$, then $f$ is a regular covering map of $U$ onto $V_{0}(r)$ ([1] p. 29). Let $U$ be a component which lies in $B$. Since $U$ is connected, there is a single annular domain $K_{j} \subset B$ with $U \subset K_{j}$. In what follows we will identify $K_{j}$ with $W=\{z: s<|z|<1\}$ and $\Gamma_{j}$ with $T$.

Given any $\varepsilon>0$ we claim that we can find a neighborhood $\Omega$ of $T$ such that if a $\varepsilon \Omega \cap W$, then $\left|f(a)-x_{0}\right|<\varepsilon$. Once we have shown this, then $f$ can be extended continuously to $T$ by setting $f(z)=x_{0}$ for $z \in T$. In this case $f$ must reduce to a constant function giving us a contradiction.

We now proceed to find the desired neighborhood $\Omega$. We may suppose that $\varepsilon<r$ and thus the conclusions above apply to the punctured disk $V_{0}(\varepsilon)$. Let $\gamma(t)$ be the circle about $x_{0}$ of radius $\varepsilon / 2$. Since $f: U \rightarrow V_{0}(\varepsilon)$ is a finite-to-one covering, a suitable multiple of $\gamma$ lifts to a closed curve $\tilde{\gamma}$ lying in $U$. Moreover, $\tilde{\gamma}$ is not null-homotopic in $W$ since otherwise $f \circ \tilde{\gamma}$ would be null-homotopic in $\mathbb{C} \backslash\left\{x_{0}\right\}$. Thus $\tilde{\gamma}$ is homotopic to a multiple of a generator of the fundamental group of $W$.

Consequently, if $\Omega$ is the unbounded component of $\mathbb{C} \backslash \tilde{\gamma}$, then $T \subset \Omega$ and $\{z:|z| \leq s\} \cap \Omega=\varnothing$. We claim that $\Omega$ is our sought-after neighborhood of $T$. Take a $\varepsilon \Omega \cap W$. We must show that $\left|f(a)-x_{0}\right|<\varepsilon$.

Let $W^{\prime}=\left\{z: s^{\prime}<|z|<1\right\}$ be chosen so that $a \notin W^{\prime}$. By the arguments given earlier, there is an $\varepsilon^{\prime}<\varepsilon$ such that $f^{-1}\left(V_{0}\left(\varepsilon^{\prime}\right)\right) \cap W \subset W^{\prime}$. Furthermore, if $\gamma^{\prime}$ is the circle about $x_{0}$ of radius $\varepsilon^{\prime} / 2$, then a suitable multiple of $\gamma^{\prime}$ lifts to a closed curve $\tilde{\gamma}^{\prime}$ lying in $W^{\prime}$ which is not null-homotopic in $W^{\prime}$. Now let $\Omega^{\prime}$ be the component of $\mathbb{C} \backslash \tilde{\gamma}^{\prime}$ which contains the origin. Then $\Omega^{\prime} \subset \Delta$. Thus, $\Omega \cap \Omega^{\prime}$ is a subset of $W$ with $\partial\left(\Omega \cap \Omega^{\prime}\right) \subset\left(\partial \Omega \cup \partial \Omega^{\prime}\right) \subset \tilde{\gamma} \cup \tilde{\gamma}^{\prime}$. Since $\left|f(z)-x_{0}\right|<\varepsilon$ on $\tilde{\gamma} \cup \tilde{\gamma}^{\prime}$ and $a \in \Omega \cap \Omega^{\prime}$, it follows by the maximum principle that $\left|f(a)-x_{0}\right|<\varepsilon$. This completes the proof.

In the next lemma important use is made of the results of " $f$-regularity". See the references [3, 4] and [9] for an exposition of this theory.

Lemma 2. Let $A$ be a function algebra on $X$. Let $W \subset M_{A}$ satisfy $\bar{W} \backslash W \subset X$ and suppose $\Psi: R \rightarrow W$ is an analytic map of $R$ onto $W$ which identifies a finite number of points. Let $F \in A$ be nonconstant on $W$ with $|F|=1$ on $X$ and suppose that the set $\left\{x \in T: \# \hat{F}^{-1}(x)<\infty\right\}$ contains a set of positive Legesgue measure. Then $\Psi$ is a proper map of $R$ onto $W$ (where $W$ has the relative topology of $M_{A}$ ) and $\tilde{F}(z)=\hat{F} \circ \Psi(z)$ is a unimodular function on $\bar{R}$. Furthermore, if $\Psi$ is a 1-1 map, then $\Psi$ is a homeomorphism.

Proof. Since $\tilde{F}$ is analytic and nonconstant on $R$ and since the topological boundary of $\hat{F}\left(M_{\mathrm{A}}\right)$ is contained in $F(X)$, it follows that $F(X)=T$ and 
$\hat{F}\left(M_{\mathrm{A}}\right)=\bar{\Delta}$. From the analyticity of $\hat{F}$ on $W$ we see that $W \cap X=\varnothing$ and $W \subset \hat{F}^{-1}(\Delta)$.

The hypotheses on $F$ imply that $\Delta$ is $F$-regular of some multiplicity $n_{0}>0$ ([9] p. 62). Since $\Psi$ identifies only a finite number of points, we may therefore conclude that there is some integer $M>0$ where $M=\max \left\{\# \tilde{F}^{-1}(z): z \in \tilde{F}(R)\right\}$. Let $V=\left\{z \in \tilde{F}(R): \# \tilde{F}^{-1}(z)=M\right\}$. Since $\tilde{F}$ is an open map, it follows that $V$ is open.

Since $\Delta$ is $F$-regular of order $n_{0}$, the set $\left\{z \in \Delta: \hat{F}^{-1}(z)<n_{0}\right\}$ is discrete in $\Delta$. Moreover, since $\Psi$ identifies only a finite number of points, it then foilows that there exists $z^{\prime} \in V$ and $g \in A$ such that $\tilde{g}=\hat{g} \circ \Psi$ separates $\tilde{F}^{-1}\left(z^{\prime}\right)$. Then for $z \in V$, let $\tilde{F}^{-1}(z)=\left\{a_{1}, \ldots, a_{M}\right\}$ and define

$$
\Phi(z)= \begin{cases}0 \quad z \in \tilde{E}(R) \backslash V \\ \prod_{i<j}\left(\tilde{g}\left(a_{i}\right)-\tilde{g}\left(a_{j}\right)\right)^{2} \quad z \in V\end{cases}
$$

Then $\Phi\left(z^{\prime}\right) \neq 0$ and $\Phi(z)$ is analytic on $V$. We claim that $\Phi$ is analytic on all of $\tilde{F}(R)$. By Rado's theorem we need prove only that $\Phi(z)$ is continuous on $\tilde{F}(R)$ ([18] p. 302).

Let $z_{0}$ be a boundary point of $V$ as a subset of $\tilde{F}(R)$. It suffices to prove that if $\left\{z_{n}\right\}$ is a sequence in $V$ which converges to $z_{0}$, then $\Phi\left(z_{n}\right)$ converges to $\Phi\left(z_{0}\right)=0$. Let $\varepsilon>0$ be given. We will find an $N$ such that if $n \geq N$, then $\left|\Phi\left(z_{n}\right)\right|<\varepsilon$.

Let $U$ be a neighborhood in $M_{\mathrm{A}}$ of $\hat{F}^{-1}\left(z_{0}\right)$ such that if $\sigma \in U$, then

$$
\min \left\{|\hat{g}(\sigma)-\hat{g}(\alpha)|: \alpha \in \hat{F}^{-1}\left(z_{0}\right)\right\}<\varepsilon^{\prime}
$$

where

$$
\varepsilon^{\prime}=\frac{1}{2} \sqrt{\frac{\varepsilon}{(2\|g\|)^{M^{2}-M-2}}} .
$$

Now as a preliminary step we wish to show that there is an integer $N^{\prime}$ such that if $n \geq N^{\prime}$, then $\Psi\left(\tilde{F}^{-1}\left(z_{n}\right)\right) \subset U$.

We suppose the contrary. Then it follows that there is a subsequence $\left\{s_{n}\right\}$ of $\left\{z_{n}\right\}$ and a sequence $\left\{w_{n}\right\}$ in $R$ with $w_{n} \in \tilde{F}^{-1}\left(s_{n}\right)$ such that $\Psi\left(w_{n}\right) \notin U$ for all $n$. Now $\left\{\Psi\left(w_{n}\right)\right\}$ is a net in $M_{A}$. Let $\Theta \in M_{A}$ be an accumulation point and let $\left\{\Psi\left(w_{j(\alpha)}\right)\right\}$ be a subnet converging to $\Theta$. The continuity of $\hat{F}$ implies that $\hat{F}\left(\Psi\left(w_{j(\alpha)}\right)\right)$ converges to $\hat{F}(\Theta)$. Since $\hat{F}\left(\Psi\left(w_{j(\alpha)}\right)\right)=\tilde{F}\left(w_{j(\alpha)}\right)=s_{j(\alpha)}$, we may conclude that $\hat{F}(\Theta)=z_{0}$. Therefore, $\Theta \in \hat{F}^{-1}\left(z_{0}\right)$ and for some $\alpha^{\prime}$ we have $\Psi\left(w_{j\left(\alpha^{\prime}\right)}\right) \in U$ giving us a contradiction.

Finally, since $\Psi$ identifies only a finite number of points, we can find $N \geq N^{\prime}$ such that if $n \geq N$, then $\Psi\left(\tilde{F}^{-1}\left(z_{n}\right)\right) \subset U$ and, additionally, so that $\Psi$ is $1-1$ when restricted to $\tilde{F}^{-1}\left(z_{n}\right)$. 
Since $z_{0} \notin V$, it follows that $\hat{F}^{-1}\left(z_{0}\right) \cap W$ contains less than $M$ points. Meanwhile, for $n \geq N$ we have $\Psi\left(\tilde{F}^{-1}\left(z_{n}\right)\right) \subset U$ and $\Psi\left(\tilde{F}^{-1}\left(z_{n}\right)\right)$ contains $M$ points. Therefore, for each $n \geq N$, there exists some $\alpha^{*} \in \hat{F}^{-1}\left(z_{0}\right) \cap W$ such that at least two points $\alpha_{n}^{\prime}$ and $\alpha_{n}^{\prime \prime} \in \Psi\left(\tilde{F}^{-1}\left(z_{n}\right)\right)$ satisfy the conditions:

$$
\begin{aligned}
& \left|\hat{g}\left(\alpha_{n}^{\prime}\right)-\hat{g}\left(\alpha^{*}\right)\right|<\varepsilon^{\prime} \\
& \left|\hat{g}\left(\alpha_{n}^{\prime \prime}\right)-\hat{g}\left(\alpha^{*}\right)\right|<\varepsilon^{\prime}
\end{aligned}
$$

which together imply that $\left|\Phi\left(z_{n}\right)\right|<\varepsilon$. Now by Rado's theorem we conclude that $\Phi(z)$ is analytic on $\tilde{F}(R)$.

Since $\Phi(z)$ is not the zero function, the zero set of $\Phi(z)$ is discrete in $\tilde{F}(R)$. In particular, the complement of $V$ is discrete in $\tilde{F}(R)$. Then as a consequence of Lemma 1 we conclude that $\tilde{F}: R \rightarrow \tilde{F}(R)$ is a proper map.

We next prove that $\Psi: R \rightarrow W$ is a proper map. Let $K \subset W$ be compact. Then $\hat{F}(K)$ is compact in $\tilde{F}(R)$. Since $\tilde{F}$ is proper, we conclude that $\tilde{F}^{-1}(\hat{F}(K))$ is compact in $R$. Then $\Psi^{-1}(K)$ is a closed subset of $\tilde{F}^{-1}(\hat{F}(K))$ and is therefore compact.

Let $\lambda \in \Gamma$ and let $\left\{z_{n}\right\}$ be a sequence in $R$ converging to $\lambda$. Since $\Psi$ is proper and $\bar{W} \backslash W \subset X$, we conclude that the accumulation points of $\left\{\Psi\left(z_{n}\right)\right\}$ are all contained in $X$. Consequently, $\operatorname{limit}_{n \rightarrow \infty}\left|\tilde{F}\left(z_{n}\right)\right|=\operatorname{limit}_{n \rightarrow \infty}\left|\hat{F} \circ \Psi\left(z_{n}\right)\right|=1$. Applying the reflection principle, we see that $\tilde{F}$ extends as a meromorphic function on the doubled surface $\hat{R}$ of $R$. It follows that $\tilde{F}$ is unimodular on $\bar{R}$.

The set $W$ is locally compact in the relative topology of $M_{A}$. To see this we note that $\tilde{F}^{-1}\left(\bar{\Delta}_{r}\right)$ is compact in $R$ where $\Delta_{r}=\{z:|z|<r\}$ and $r<1$. Hence $\Psi\left(\tilde{F}^{-1}\left(\bar{\Delta}_{r}\right)\right)$ is compact in $W$. Since $\hat{F}^{-1}\left(\Delta_{r}\right) \cap W \subset \Psi\left(\tilde{F}^{-1}\left(\bar{\Delta}_{r}\right)\right)$, it follows that $W$ is locally compact. Consequently, if we assume in addition that $\Psi$ is $1-1$, then $\Psi$ is a homeomorphism ([14] p. 285). This completes the proof.

The remarks above contain a proof of the following Lemma.

Lemma 3. Let $A$ be a function algebra on $X$. Let $W \subset M_{A}$ satisfy $\bar{W} \backslash W \subset X$ and suppose $\Psi: R \rightarrow W$ is a proper analytic map of $R$ onto $W$. Then if $g \in A$ satisfies $|g|=1$ on $X$, then $\tilde{g}(z)=\hat{g} \circ \Psi(z)$ is a unimodular function on $\bar{R}$ or $\tilde{g}$ is a constant function.

The assumption of continuity for an analytic map $\Psi$, rather than assuming further that $\Psi$ is also a proper map, is natural since various embedding theorems which give analytic structure to certain types of subsets $W$ in $M_{A}$ produce a map $\Psi: R \rightarrow W$ which need only be continuous. (See [8] p. 154-161 and [18] p. 170-187 for a discussion of analytic structure in Gleason parts. This topic will be developed in §4.) Lemma 2 then gives conditions on $A$ and $W$ which imply that an analytic mapping $\Psi: R \rightarrow W$ is proper. The papers [11] and [13] discuss this topic and related problems.

Lemma 4. Let $A$ be a function algebra on $X$. Let $W \subset M_{A}$ satisfy $\bar{W} \backslash W \subset X$ and suppose $\Psi: R \rightarrow W$ is an analytic mapping of $R$ onto $W$ which identifies a 
finite number of points. Assume that $S=\{g \in A:|g|=1$ on $X\}$ separates points on $X$ and that there is $F \in S$, nonconstant on $W$, such that $\left\{x \in T: \# \hat{F}^{-1}(x)<\infty\right\}$ contains a set of positive Lebesgue measure. Then

(i) $\Psi$ extends continuously to $\bar{R}$ and maps $\Gamma$ into $X$.

(ii) If $f \in A$, then $\tilde{f}=\hat{f} \circ \Psi \in A(R)$.

(iii) If $L=\{(a, b) \in \bar{R} \times \bar{R}: a \neq b, \Psi(a)=\Psi(b)\}$, then $(a, b) \in L$ if and only if $\tilde{f}(a)=\tilde{f}(b)$ for all $f \in A$.

Proof. By the preceding lemmas it follows that $\Psi$ is proper. We now prove (i). Let $\lambda \in \Gamma$ and let $\left\{a_{n}\right\} \subset R$ converge to $\lambda$. We may assume that $\left\{a_{n}\right\}$ does not meet the set of points identified by $\Psi$. Since $\Psi$ is proper and $\bar{W} \backslash W \subset X$, it follows that the accumulation points of $\left\{\Psi\left(a_{n}\right)\right\}$ are contained in $X$. Suppose there exist distinct accumulation points $x$ and $y$. By assumption there is $g \in S$ so that $g(x) \neq g(y)$.

Let $\left\{\alpha_{\beta}\right\}$ be a subnet of $\left\{\Psi\left(a_{n}\right)\right\}$ which converges to $x$. By continuity $\hat{g}\left(\alpha_{\beta}\right)$ converges to $\hat{g}(x)$. Since $\hat{g}\left(\alpha_{\beta}\right)=\tilde{g}\left(\Psi^{-1}\left(\alpha_{\beta}\right)\right)$ and $\tilde{g} \in A(R)$ by Lemma 3 , we may conclude that $\hat{g}(x)=\tilde{g}(\lambda)$. Likewise, we may show that $\hat{g}(y)=\tilde{g}(\lambda)$. Hence, we must have $x=y$. We let $\Psi(\lambda)$ denote the unique accumulation point of $\left\{\Psi\left(a_{n}\right)\right\}$. If $\left\{b_{n}\right\} \subset R$ is another sequence which converges to $\lambda$, a similar argument will show that $\Psi(\lambda)$ is the only accumulation point of $\left\{\Psi\left(b_{n}\right)\right\}$.

We use $\Psi(\lambda)$ to extend $\Psi$ to $\Gamma$. We have $\Psi(\Gamma) \subset X$ and a straightforward argument shows that $\Psi$ is continuous on $\bar{R}$. The proofs of (ii) and (iii) are now immediate.

Proof of Theorem 1. Since $\bar{W} \backslash W=X$, we conclude that $\Psi(\Gamma)=X$. Let $\tilde{\Psi}: A \rightarrow A(R)$ be defined by $\tilde{\Psi}(f)=\hat{f} \circ \Psi$. Then $\Psi$ is an isomorphism of $A$ onto $\tilde{\Psi}(A)$. Set $A^{\prime}=\tilde{\Psi}(A)$.

Let $g \in S$ be nonconstant. Then $\tilde{g}$ is unimodular of some order $n$. Since $\Psi$ identifies only finitely many points of $R$, we can find some $w_{0} \in \Delta$ and $f \in A$ such that $\tilde{f}$ separates $\tilde{g}^{-1}\left(w_{0}\right)$. Let $\tilde{g}^{-1}(w)=\left\{z_{1}, \ldots, z_{n}\right\}$ (including repetitions) for $w \in \mathbb{C}$. Set $w \in \bar{\Delta}$ and let $z_{1}=z$. Then $\Theta(z)=\prod_{i=2}^{n}\left(\tilde{f}(z)-\tilde{f}\left(z_{i}\right)\right) \in A(R)$ and $\Theta(z) \not 0$. In particular, there is some $t \in T$ such that $\tilde{f}$ separates the $n$ distinct points of $\tilde{g}^{-1}(t)$. By (iii) of Lemma 4 we conclude that $\Psi$ is $1-1$ on $\tilde{g}^{-1}(t)$.

Since $S$ separates points on $X$, there is a polynomial $Q$ in elements of $S$ such that $Q$ separates the points of $\Psi\left(\tilde{g}^{-1}(t)\right)$. Note that both $\tilde{g}$ and $\tilde{Q}$ extend meromorphically to $\hat{R}$. It follows that $\Omega(z)=\prod_{i=2}^{n}\left(\tilde{Q}(z)-\tilde{Q}\left(z_{i}\right)\right)$ is analytic on a neighborhood of $\bar{R}$ and $\Omega(z) \not \equiv 0$. In this case the singular part of $\Omega(z)$ as an element of $A(R)$ in the sense of [16] is zero. This property of $\Omega(z)$ is evident if $\Omega(z)$ has no zeros on $\Gamma$ and can be established by localization and the use of the inner-outer factorization for $A(\Delta)$ if $\Omega(z)$ has zeros on $\Gamma$.

If $J=\left\{f \in A(R): f A(R) \subset A^{\prime}\right\}$, then $J$ is a closed ideal of $A(R)$ with $J \subset A^{\prime}$ and $\Omega(z) \in J([12])$. Since $\Omega(z)$ has zero singular part and only a finite number 
of zeros in $R$, it follows that $J$, and hence $A^{\prime}$, has finite codimension in $A(R)$ ([16], Theorem I and [15], Lemma 2.5).

We recall that $M_{A(R)}=\bar{R}([2])$. The maximal ideal space of $A^{\prime}$ is obtained by identifying the points of $\bar{R}$ which are identified by $\Psi$ ([7], Theorem 9.8). We see then that $\tilde{\Psi}^{*}: M_{\mathrm{A}^{\prime}} \rightarrow M_{\mathrm{A}}$ defined by $\tilde{\Psi}^{*}(\sigma)(f)=\sigma(\tilde{\Psi}(f))$ for $\sigma \in M_{\mathrm{A}^{\prime}}$ and $f \in A$ is a homeomorphism and $M_{A}=\tilde{\Psi}^{*}\left(M_{A^{\prime}}\right)=\Psi(\bar{R})=\bar{W}$. Therefore, $\bar{W}=$ $M_{\mathrm{A}}$ as required. That completes the proof.

ReMARK. In the proof of Theorem 1 we constructed two functions $\tilde{g}$ and $\tilde{Q}$ that separate all but a finite subset of $\bar{R}$. Hence, according to Lemma 4 (iii), we may conclude that the set $L=\{(a, b) \in \bar{R} \times \bar{R}: a \neq b, \Psi(a)=\Psi(b)\}$ is finite. This remark will be used in $\$ 4$.

$\S 4$ Analytic maps and Gleason parts. In this section we apply Gamelin's theorem on embedding analytic structure in certain subsets of the maximal ideal space of a function algebra to Theorem 1 to obtain entirely "internal" conditions on $A$ which imply that $A$ is isomorphic to a finite codimensional subalgebra of $A(R)$. We will use standard definitions and results in function algebras which are found in the references [8] or [18].

Theorem (Gamelin [8] p. 161 and [7]). Let $A$ be a function algebra on $X$. Suppose the set of representing measures supported on $X$ for $\phi \in M_{A}$ is finite dimensional and that $\phi$ has a unique logmodular measure on $X$. Suppose the Gleason part $P$ which contains $\phi$ contains more than one point. Then there is a 1-1 analytic mapping $\Psi$ of $R$ onto $P$.

The mapping $\Psi$ need not be a homeomorphism nor even a proper map (see [5] p. 251 and [13] p. 1095). By combining Theorem 1 and Gamelin's theorem we obtain the following result:

Theorem 2. Let $A$ be a function algebra on $X$. Assume that

(i) There is $\phi \in M_{A}$ such that the set of representing measures on $X$ for $\phi$ is finite dimensional and that $\phi$ has a unique logmodualr measure $m$ on $X$. Furthermore, suppose that the Gleason part $P$ which contains $\phi$ satisfies $\bar{P} \backslash P=$ $X$.

(ii) The set $S=\{g \in A:|g|=1$ on $X\}$ separates points on $X$ and there is $F \in S$, nonconstant on $X$, such that $E=\left\{x \in T: \# \hat{F}^{-1}(x)<\infty\right\}$ contains a set of positive Lebesgue measure in $T$.

Then there is a finite set $L$ contained in $\{(a, b) \in \Gamma \times \Gamma: a \neq b\}$ such that $A$ is isomorphic to the algebra $A^{\prime}=\{f \in A(R): f(a)=f(b)$ for $(a, b) \in L\}$.

Before beginning the proof of Theorem 2, we will briefly discuss the analytic mapping of Gamelin's theorem as well as the necessary background from function algebra theory. If $\phi \in M_{A}$ we will let $M_{\phi}(X)$ denote the set of representing measures supported on $X$ for $\phi$. If $M_{\phi}(X)$ is finite dimensional 
and if $\phi$ has a unique logmodular measure on $X$, then all the representing measures for $\phi$ are mutually absolutely continuous ([7] p. 129-130). Now let $\phi$ have finite dimensional $M_{\phi}(X)$ with unique logmodular measure $m$ on $X$. If $\Theta \in M_{\mathrm{A}}$ belongs to the same Gleason part as $\phi$, then $M_{\Theta}(X)$ is also finite dimensional and each $\mu \in M_{\Theta}(X)$ is mutually absolutely continuous with $m$ ([8] p. 143).

We now fix $\phi \in M_{\mathrm{A}}$ such that $M_{\phi}(X)$ is finite dimensional and contains a unique logmodular measure $m$ and we suppose that the part $P$ containing $\phi$ contains more than one point. We let $H^{\infty}(m)$ denote the weak-star closure of $A$ in $L^{\infty}(m)$. Let $\iota: A \rightarrow H^{\infty}(m)$ be the inclusion map and let the continuous map $\iota^{*}: M_{H^{\circ}(m)} \rightarrow M_{A}$ be given by $\iota^{*}(\Theta)(f)=\Theta(\iota(f))$ and $f \in A$ and $\Theta \in M_{H^{*}(M)}$.

Each $\Theta \in P$ extends to a homomorphism $\Theta^{\prime} \in M_{H^{\infty}(m)}$ by setting $\Theta^{\prime}(f)=\int f d \mu$ for $f \in H^{\infty}(m)$ where $\mu \in M_{\Theta}(X)$. Let $\Pi$ be the part of $M_{H^{\infty}(m)}$ which contains $\phi^{\prime}$. If $\Theta^{\prime} \in \iota^{*-1}(P)$, then the representing measures for $\Theta^{\prime}$ can be identified with $M_{\Theta}(X)\left([8]\right.$, p. 102). By a short argument it follows that $\iota^{*}$ is a 1-1 map of $\Pi$ onto $P$. Furthermore, if $f_{n} \in A$ converges weak-star to $f \in H^{\infty}(m)$, then $\iota\left(f_{n}\right)$ converges pointwise to $\hat{f}$ on $\Pi$. (cf [18] p. 183-184).

Gamelin's theorem produces a $1-1$ analytic map $\chi$ from $R$ onto $\prod$ with respect to the algebra $H^{\infty}(m)$. Then $\Psi=\iota^{*} \circ \chi$ is a $1-1$ analytic map from $R$ onto $P$ for the algebra $A$. Notice that $\widehat{\iota(f)} \circ \chi=\hat{f} \circ \Psi$ for $f \in A$. We remark that if a bounded sequence $\left\{f_{n}\right\}$ in $A$ converges weak-star to $f \in H^{\circ}(m)$, then $\iota \widehat{\left(f_{n}\right) \circ \chi}$ converges uniformly on compact subsets of $R$ to $\hat{f} \circ \chi$.

Proof of Theorem 2. Gamelin's theorem supplies a 1-1 analytic map $\Psi$ of $R$ onto $P$, and this map extends continuously to $\bar{R}$ to map $\Gamma$ onto $X$ according to Theorem 1. The map $\tilde{\Psi}: A \rightarrow A(R)$ defined by $\tilde{\Psi}(f)=\hat{f} \circ \Psi$ is an isomorphism of $A$ onto $\tilde{\Psi}(A)$. Set $A^{\prime}=\tilde{\Psi}(A)$. Let $L=\{(a, b) \in \bar{R} \times \bar{R}: a \neq b$ and $\Psi(a)=\Psi(b)\}$. By the remark following the proof of Theorem 1 we know that $L$ is finite. Also, since $\Psi$ restricted to $R$ is $1-1$, we conclude that $L \subset \Gamma \times \Gamma$.

Define $B^{\prime}=\{f \in A(R): f(a)=f(b)$ if $(a, b) \in L\}$. Then $A^{\prime} \subset B^{\prime}$. To complete the proof we must show that $A^{\prime}=B^{\prime}$. Assume instead that $A^{\prime}$ is properly contained in $B^{\prime}$. By Theorem 1 we know that $A^{\prime}$ has finite codimension in $A(R)$, and hence, in $B^{\prime}$. Consequently, there is a nonzero continuous point derivation $D$ on $B^{\prime}$ such that $A^{\prime} \subset \operatorname{ker} D$ ([7], Theorem 9.8).

Since $M_{A(R)}=\bar{R}$, the maximal ideal space for $B^{\prime}$ is the quotient space obtained by identifying the points of $\bar{R}$ which are identified by $\Psi$. Each level set of the restriction of $\Psi$ to $\Gamma$ is a peak set for $B^{\prime}$ and such sets are peak points when $B^{\prime}$ is regarded as a function algebra on $M_{B^{\prime}}$. It follows that $D$ must be a point derivation at some point $s \in R$ ([5], Corollary 1.6.7). Consequently, $A^{\prime} \subset \operatorname{ker} D=M_{s}^{2}+\mathbb{C}$ where $M_{s}=\left\{f \in B^{\prime}: f(s)=0\right\}$ and $M_{s}^{2}=\left\{f g: f\right.$ and $\left.g \in M_{s}\right\}$. As a result we conclude that if $h \in A$, then $d \tilde{h}(s)=0$ where $\tilde{h}=\hat{h} \circ \Psi$ and $d \tilde{h}(s)$ is the differential of $\tilde{h}$ at $s$. 
According to hypothesis there is a unique logmodular measure $m$ on $X$ for $\phi \in P$. Let $\Psi^{-1}(\phi)=z_{0}$. There is a logmodular measure $m_{0}$ for $z_{0}$ supported on $\Gamma$ for the algebra $A(R)$ ([8] p. 33 and 110). We may define a Borel measure $m^{\prime}$ on $X$ by setting $m^{\prime}(K)=m_{0}\left(\Psi^{-1}(K)\right)$ for Borel sets $K \subset X$. Then it follows that $m^{\prime}$ is a logmodular measure for $\phi$. Consequently, $m^{\prime}=m$.

Let $B=\left\{f \in C\left(M_{A}\right): f \circ \Psi \in B^{\prime}\right\}$. Then $m$ is multiplicative with respect to $B$. Let $H^{\infty}(B)$ be the weak-star closure of $B$ in $L^{\infty}(m)$. Then $H^{\infty}(m) \subset H^{\infty}(B)$. According to a maximality theorem ([8] p. 115) we have $H^{\infty}(m)=H^{\infty}(B)$.

If $f \in H^{\infty}(m)$, then there is $\left\{f_{n}\right\} \subset A$ such that $\left\|f_{n}\right\| \leq\|f\|$ and $f_{n}$ converges to $f$ a.e. $[m]\left([10]\right.$, p. 137 and p. 149). In this case $\iota\left(f_{n}\right) \circ \chi$ converges uniformly on compact subsets of $R$ to $\hat{f} \circ \chi$. As a result $d(\hat{f} \circ \chi)(s)=0$ for all $f \in H^{\infty}(m)$. However, it is easy to construct a $G \in B$ such that for $g=G \circ \Psi \in B^{\prime}$ we have $d g(s) \neq 0$. Furthermore, $\iota(G) \in H^{\infty}(m)$ and $\iota(G) \circ \chi(z)=g(z)$. Consequently, $d(\iota(G) \circ \chi)(s) \neq 0$ giving a contradiction. This completes the proof.

Let $A$ be a function algebra on $X$. We say that $A$ is a Dirichlet algebra on $X$ if $\{\operatorname{Re}(f): \in A\}$ is uniformly dense in the set of real-valued continuous functions on $X$. An algebra $A$ is a Dirichlet algebra on $X$ if and only if there are no nonzero real annihilating measures of $A$ supported on $X$.

Let $A^{\perp}=\left\{\mu: \mu\right.$ is a finite regular Borel measure on $X$ such that $\int f d \mu=0$ for all $f \in A\}$.

Corollary 1. In addition to the assumptions of Theorem 2 suppose that $\phi \in M_{A}$ has a unique representing measure supported on $X$. Then $A$ is a Dirichlet algebra on $X$.

Proof. The Riemann surface $R$ of Gamelin's theorem is now the open unit disk $\Delta$. ([8], p. 158) and $\Gamma$ becomes $T$. Let $\mathscr{S}=\{K \subset T: \Psi(K)$ is Borel $\}$. It is straightforward to show that $\mathscr{S}$ is a $\sigma$-algebra which contains the Borel sets. Suppose that $\mu \in A^{\perp}$ is a nonzero real measure on $X$. Let $E=\{t \in T$ : there is $t^{\prime} \neq t$ with $\left.\Psi\left(t^{\prime}\right)=\Psi(t)\right\}$. By Theorem 2 we know that $E$ is a finite set. Now for $t \in E$ we let $E_{t}=\left\{t^{\prime} \in E: \Psi\left(t^{\prime}\right)=\Psi(t)\right\}$. Using Theorem 2 we see that $\Psi\left(E_{t}\right)$ is a peak point for the function algebra $A$ and therefore $\mu\left(\Psi\left(E_{t}\right)\right)=0$. Furthermore, we see that $|\mu|(\Psi(E))=0$. It now follows that we may define a finite regular Borel measure $\tilde{\mu}$ on $T$ by setting $\tilde{\mu}(K)=\mu(\Psi(K))$ for Borel sets $K \subset T$. Then $\tilde{\mu}$ is real and $\tilde{\mu}$ annihilates $A^{\prime}$.

Let $f \in A^{\prime}$ be an outer function which vanishes precisely on $E$. Then $f A(\Delta) \subset A^{\prime}$ and so $f \tilde{\mu} \in A(\Delta)^{\perp}$. By the F. and M. Riesz theorem there is $h \in H_{0}^{1}(\Delta)$ such that $f \tilde{\mu}=h \lambda$ where $\lambda$ is Lebesgue measure on $T$. As a result we have $\tilde{\mu}=(h / f) \lambda+\left.\tilde{\mu}\right|_{E}$. Since $|\tilde{\mu}|(E)=0$, we obtain $\tilde{\mu}=(h / f) \lambda$. Now $h / f$ is real a.e. $[\lambda]$ and $h / f \in L^{1}(T)$. But then $h / f \in H^{1}(\Delta)$ ([6] p. 28). But a function in $H^{1}(\Delta)$ which is real a.e. must be constant. Since $0=\int d \tilde{\mu}=\int(h / f) d \lambda$, we see that $h / f=0$ a.e. $[\lambda]$. Therefore, $\tilde{\mu}$ is the zero measure and it follows that $\mu$ is the zero measure on $X$. This proves the Corollary. 
Remark. One may check that the algebra $A=\{f \in A(\Delta): f(1)=f(-1)\}$ regarded as a function algebra on the set $X$ obtained from $T$ by identifying the points 1 and -1 satisfies the hypotheses of Theorem 2. (A point $z \in \Delta$ has a unique representing measure on $X$ since $A$ is Dirichlet on $X$ as shown by the proof of Corollary 1.) As a result we see that the hypotheses of Theorem 2 do not allow us to capture the entire algebra $A(R)$ through the isomorphism $\tilde{\Psi}$. A condition which implies that $\tilde{\Psi}$ maps $A$ onto $A(R)$ is given in the Corollary below.

COROllary 2. In addition to the assumptions of Theorem 2 suppose there is a positive integer $n$ such that $F: X \rightarrow T$ is precisely an $n$-to-one map. Then $A$ is isomorphic to $A(R)$.

Proof. We will prove that $\Psi: \bar{R} \rightarrow M_{\mathrm{A}}$ is a 1-1 map which will establish the Corollary. The order of the unimodular function $\tilde{F}=\hat{F} \circ \Psi$ is at most $n$ ([9] p. 62). If $t \in T$, then $\Psi\left(\tilde{F}^{-1}(t)\right) \subset \hat{F}^{-1}(t)$. Since $\Psi$ maps $\Gamma$ onto $X$, we may conclude that $\tilde{F}$ has order $n$ and that $\Psi$ is $1-1$ on $\tilde{F}^{-1}(t)$. By Lemma 4 (iii), we conclude that $\Psi$ is $1-1$ on $\Gamma$. Consequently, using Theorem 2 we see that $\Psi$ is $1-1$ on all of $\bar{R}$. This completes the proof.

\section{REFERENCES}

1. L. Ahlfors and L. Sario, Riemann surfaces, Princeton University Press, Princeton, N.J., 1960.

2. R. Arens, The closed maximal ideals of algebras of functions holomorphic on a Riemann surface, Rend. Cir. Mat. di Palermo (2) 7 (1958), 245-260.

3. J.-E. Björk, Analytic structures, Papers from the Summer Gathering on Functional Algebras at Aarhus, July (1969), 19-28.

4. J.-E. Björk, On analytic structure in the maximal ideal space of a function algebra, Papers from the Summer Gathering on Function Algebras at Aarhus, July (1969), 29-35.

5. A. Browder, Introduction to function algebras, W. A. Benjamin, New York, 1969.

6. P. L. Duren, Theory of $H^{P}$ spaces, Academic Press, New York, 1970.

7. T. W. Gamelin, Embedding Riemann surfaces in maximal ideal spaces, J. Functional Analysis 2 (1968), 123-146.

8. T. W. Gamelin, Uniform algebras, Prentice-Hall, Englewood Cliffs, N.J., 1969.

9. T. W. Gamelin, Polynomial approximation on thin sets, Symposium on Several Complex Variables, Park City, Utah (1970), Lecture notes in mathematics 184, Springer Verlag, Berlin, 1971.

10. T. W. Gamelin and G. Lumer, Abstract Hardy spaces and universal Hardy class, Advances in Math. 2 (1968), 118-174.

11. K. Kiski, Homeomorphism between the open unit disk and a Gleason part, J. Math. Soc. Japan 27 (1975), 467-473.

12. B. Lund, Subalgebras of finite codimension in the algebra on analytic functions on a Riemann surface, Pacific J. Math. 51 (1974), 495-497.

13. B. Lund, Analytic embeddings in logmodular algebras, Indiana Univ. Math. J. 24 (1975), 1093-1098.

14. J. R. Munkres, Topology, Prentice-Hall, Englewood Cliffs, N.J., 1975.

15. A. Read, A converse of Cauchy's theorem and applications to extremal problems, Acta Math. 100 (1958), 1-22.

16. C. M. Stanton, The closed ideals of a function algebra, Trans, Amer. Math. Soc. 154 (1971), 289-300. 
17. E. L. Stout, On some algebras of analytic functions on finite open Riemann surfaces, Math. Z. 92 (1966), 366-379.

18. E. L. Stout, The theory of uniform algebras, Bogden and Quigley, Tarrytown-on-Hudson, N.Y., 1971.

DEPARTMENT OF MATHEMATICS

UNIVERSITY OF NEW BRUNSWICK

Fredericton, N. B., CANADA 